Psychological Medicine, 1991, 21, 279-285

Printed in Great Britain

EDITORIAL

\title{
Doubt about evidence for a schizophrenia gene on chromosome $5^{1}$
}

The report that DNA markers on chromosome 5 showed overwhelming evidence of linkage to a gene for susceptibility to schizophrenia (Sherrington et al. 1988) has naturally aroused widespread interest. The failure to confirm this finding (Kennedy et al. 1988; St Clair et al. 1989; DeteraWadleigh et al. 1989) has hardly dampened optimism or deterred advocates from ambitious plans for building on this foundation. However, the recent withdrawal (Kelsoe et al. 1989) of a similar apparently secure claim to have localized a genetic basis for bipolar affective disorder by linkage analysis (Egeland et al. 1987) should give cause for further scrutiny of the basis for believing that linkage of a marker to a locus related to schizophrenia has been found on chromosome 5 .

\section{LINKAGE ANALYSIS}

\section{The basis of linkage}

Linkage arises from the fact that offspring inherit genetic determinants from each parent in the form of blocks of genes, linearly arranged as chromosomes, in the order in which they occur in the donating parent. These may be interrupted at points by the exchange of material (crossover or recombination) between these homologous chromosomes at meiosis.

Linkage is demonstrated when genes which are adjacent in a parent can be shown to have remained so in a proportion of offspring substantially more than the $50 \%$ expected from random assortment. Thus, the offspring can be classified as recombinant or non-recombinant in respect of a neighbouring pair of genes.

The extent to which the ratio of recombinants to non-recombinants in a pair of genes among the individuals of an extended family departs from the fifty-fifty ratio of random assortment gives a measure of both the likelihood of linkage and its closeness between two loci. As it is not always possible to deduce recombinants and non-recombinants due to incompleteness of some families, Morton (1955) devised a method by which the proportion of recombinants is assumed and the 'likelihood ' of this being consistent with the data calculated. This 'likelihood' is then compared to the likelihood expected in the absence of linkage and the ratio of these two numbers is the likelihood ratio or 'odds ratio'. Its logarithm, or 'lod', has the advantage that it can be added between families. With close linkage each informative meiosis contributes $\log 2$ or about a third of a lod. A lod of 3 is equivalent to about ten meioses without a recombinant and, as with ten successive heads in a coin-tossing, this gives ground for suspicion that linkage has taken place (or that a double-headed coin was used!).

\section{Obstacles to demonstrating linkage of DNA markers to a putative gene for schizophrenia}

Linkage was first demonstrated in 1908 in flowering plants (Bateson \& Punnet, 1908); then in insects (the fruit fly) in 1912 (Morgan, 1912) and in vertebrates (mouse) in 1915 (Haldane et al.). There was a gap of over twenty years before linkage with a disease locus (haemophilia on the X chromosome) was first demonstrated in man (Bell \& Haldane, 1937) and another fifteen years before the first demonstration of linkage not involving the $\mathrm{X}$ chromosome (myotonic dystrophy) (Mohr, 1954). This delay reflects the comparatively limited number of living generations it is possible to survey in man. The marker used by Mohr, secretor status, which has been called an 'honorary blood group' (Race \& Sanger, 1975), illustrates an important factor which facilitated the demonstration of human

' Address for correspondence: Dr D.C. Watt, 7 Churchway, Stone, Aylesbury, Bucks, HP17 8RG. 
linkage. Blood groups were pre-eminent as biological markers in that they were accessible, showed clear Mendelian inheritance and full penetrance with the phenotype unmistakably and unvaryingly expressed, unmodified by age, disease or environmental conditions. They are now largely replaced by restriction fragment length polymorphisms (RFLP). Schizophrenia, by contrast, is variable, has no diagnostic test and diagnosis is totally dependent on clinical judgement. Its boundaries are unclear so that it is easily confused with other conditions and it is markedly susceptible to environmental influence.

Linkage analysis used to elucidate the genetic mechanism of schizophrenia requires an assumption that a gene strongly predisposing to the disorder exists so that linkage to a marker can be sought. Blood groups and HLA antigens have been employed in this way with negative or, at most, tenuous results (Sturt \& McGuffin, 1985). A fresh impetus to linkage analysis was provided by the discovery of restriction fragment length polymorphisms. Here lengths of DNA are cut into short segments by restriction enzymes and identified by radioactive probes which will adhere only to a specific complementary segment of DNA. These have the advantages that they, compared with biological markers such as blood groups, are often more polymorphic and are indefinitely numerous with a heterozygote distinct from both homozygotes. By suitable labelling they can be mapped and thus indicate a locus close to the locus responsible for a genetic disease.

\section{CHROMOSOME 5 AND SCHIZOPHRENIA}

The starting point for interest in chromosome 5 as the location for a schizophrenia-susceptibility gene is the report by Basset (1989) of a proband and his maternal uncle, both of whom had a psychiatric disorder and also an unusual facies. These features were not present in the five other first degree relatives. Both affected individuals were found to have an extra copy of a small portion of chromosome 5 (q11-13) translocated to chromosome 1 with a reciprocal exchange between chromosome 5 and 1 in the mother of the proband.

The mother was affected with neither the facial nor psychiatric phenotypic abnormality suggesting that the duplicated chromosomal material, rather than its rearrangement, was the cause of the psychiatric disorder and dysmorphic features in her son and in her brother. The fact that chromosome $5 q$ contains the gene encoding the lymphocyte glucocortisoid receptor, which could be implicated in predisposing to schizophrenia, lent support to the view that this chromosome area merited investigation as a candidate schizophrenia-susceptibility gene.

\section{LINKAGE ANALYSIS IN FAMILIES MANIFESTING SCHIZOPHRENIA}

In order to explore this possibility Sherrington et al. (1988) carried out a linkage analysis on 7 schizophrenic families with several affected members using as probes two restriction fragment length polymorphisms (RFLP) known to adhere to chromosome 5 at the glucosteroid receptor locus. The first probe defined three allelic fragments and the second two. The aim of the investigation was to compare the degree to which particular fragments segregated with the postulated locus predisposing to schizophrenia on chromosome 5 in schizophrenic subjects as opposed to non-schizophrenic subjects: i.e. to detect linkage between the probe locus and the postulated schizophrenia-susceptibility gene locus. The authors claimed to have found such a linkage and thus to have 'demonstrated strong evidence of a single gene located on chromosome 5 in the causation of schizophrenia'. Three attempts to confirm this result using the same method have been reported (Kennedy et al. 1988; St Clair et al. 1989; Detera-Wadleigh et al. 1989). None has given evidence of linkage.

\section{EXPLANATIONS FOR CONTRADICTORY RESULTS}

Commentators attempting to reconcile the contradictory claims for linkage of a marker to a schizophrenia susceptibility locus on chromosome 5 have pointed to a number of possible 
confounding factors: that the gene on chromosome 5 may not exist or may be of low penetrance in the families involved in the repeat studies (Lander, 1988; Byerley, 1989); expression of the gene may be greatly influenced by the environment (Lander, 1988); that it may be an error to assume dominant transmission, which may be recessive (Byerley, 1989). An implication of these suggestions is variously that in the 5 families participating in the investigation of Sherrington et al. (1988) a highly penetrant schizophrenia-susceptibility gene was segregating but was not present in the (equally schizophrenic) families of the three repeat investigations. The probability against such selection occurring by chance in the families of Sherrington et al. (1988) is well over 1000 to 1. Further, the implication that possible error in the mode of transmission or degree of penetrance assumed is attributable only to the three repeat studies is reminiscent of the Scots saying 'All out of step but our Jimmie'.

A universally proposed explanation, however, is that schizophrenia is genetically heterogeneous, in that many alleles at several loci lead to predispositions which become manifest in appropriate environments. Proceeding on this assumption, Detera-Wadleigh et al. (1989) state that 'the resolution of the varying linkage results may have to await study of a large series of families'. Byerley (1989) proposes that through technology now coming into use a mutant allele may be systematically searched for using 200-400 markers and 'within the next few years the goal is to construct a one-centimorgan linkage map which will greatly facilitate gene identification...'. This is an undertaking involving vast resources with no guarantee, or even expectation, of success. Therefore, before allocating precious resources on this scale further scrutiny is needed of the evidence on which a locus for a schizophrenia-susceptibility gene on chromosome 5 is claimed.

\section{METHODS AND RESULTS OF SHERRINGTON et al. (1988)}

Difficulties inherent in searching for linkage of a marker to schizophrenia arise from the general problem of diagnosis in psychiatry which is acute in schizophrenia and from the pattern of transmission of schizophrenia within families. Thus, Sherrington et al. (1988) for their analysis, divide psychiatrically affected subjects into three categories: (1) cases diagnosed as schizophrenia, as schizophreniform disorder and as unspecified functional psychosis; (2) cases diagnosed as schizoid personality disorder; (3) miscellaneous psychiatric disorders such as depression, alcoholism, drug misuse, phobias. The definitions of all these disorders are those provided by the Diagnostic and Statistical Manual of Psychiatric Disorders, 3rd edn (DSM-III) (APA, 1980). Separate linkage analyses were carried out for category 1, categories 1 plus 2 and categories 1 plus 2 plus 3 . The lods found for each analysis were $3.22,4.33$ and 6.49 respectively.

\section{PROBLEMS IN CHECKING RESULTS REPORTED BY SHERRINGTON et al (1988)}

Any attempt to check the basis for the results reported by Sherrington et al. (1988) is confronted with obstacles of two kinds. The first are the difficulties, already outlined, inherent in demonstrating linkage in schizophrenia. The second is concerned with the presentation of method and results in this paper which shows certain omissions and inconsistencies. Seven pedigrees were reported in the text, but only six were shown. The key to the symbols used in the pedigrees to differentiate the psychiatric disorders which the authors have included in their analyses is misleading in several respects so that it is impossible to know unambiguously what some symbols represent. For instance, if those shown in the key to Fig. 1 of their paper are numbered 1-10 from top to bottom:

(a) A female individual is represented by a square with corners cut (information from $\mathrm{Dr}$ H. Gurling) e.g. F36, II, 1 and 2. Symbol 4 therefore indicates a female suffering from schizophrenia, schizophreniform disorder or unspecified functional psychosis, not schizoid personality as shown in the key. The symbol for schizoid personality appears in the pedigrees only e.g. at F36, II, 1 and III, 2 and 3) and not in the key;

(b) The symbols for minor depressive disorder (6) and drug use disorder (9) are indistinguishable;

(c) The symbol shown in the pedigrees at F27, II, 3 and F40, II, $2+3$ does not appear in the key. 
Table 1. Number shown in the text in each diagnostic category compared with the number shown in the pedigrees (Sherrington et al. 1988)

\begin{tabular}{|c|c|c|c|c|c|}
\hline & \multicolumn{5}{|c|}{ Number of marker-typed individuals } \\
\hline & Affected & Unaffected & $\begin{array}{l}\text { SCZ,SCZFRM } \\
\text { unspecified }\end{array}$ & $\begin{array}{c}\text { SCZoid } \\
\text { personality }\end{array}$ & $\begin{array}{c}\text { 'Fringe' } \\
\text { pheno- } \\
\text { type }\end{array}$ \\
\hline Pedigree & 58 & 78 & 42 & 6 & 10 \\
\hline Text & 54 & 50 & 39 & 5 & 10 \\
\hline
\end{tabular}

SCZ, Schizophrenia; SCZFRM, schizophreniform; SCZoid, schizoid.

Even when corrections are made in accordance with information from the authors, discrepancies appear between the numbers of marker-typed individual assigned to each diagnostic category in the text and those in the pedigrees, as shown in Table 1.

In calculating lods the basic datum is recombination frequency. Recombination is more frequent in females than in males in most of the genome and allowance is often made for the proportion of females included in the families analysed. In the pedigrees of Sherrington et al. (1988) the sex of subjects is falsified (in order to avoid family members recognizing themselves if they read the published pedigrees) so that it is impossible for a reader to know what allowance has been made for this factor or to check the lods. (The sex of subjects was kindly supplied to the present authors by $\mathrm{Dr} \mathrm{H}$. Gurling for six of the seven pedigrees.) Where marker typing is not possible for a subject who is a parent (e.g. having died) the marker genotype can sometimes be inferred if the typing of the spouse and of offspring is known. In the first generation of pedigree 20, a deceased spouse is shown with marker typing inferred, for which, however, no basis is shown in the pedigree data. If the marker data for this subject are not known, it is possible for the number of recombinants in this family to be 3 instead of 0 as is assumed.

Although schizophrenia is clearly familial, its pattern of segregation does not conform closely to any Mendelian pattern. For linkage analysis, however, segregation of a single gene showing a dominant pattern has usually been assumed, i.e. that affected persons have an affected parent and the proportion of affected offspring from an affected parent approximates to $50 \%$. However, transmission through two unaffected parents is usual in schizophrenic pedigrees, indeed most schizophrenics have non-schizophrenic parents and children. Thus, sporadic (i.e. non-genetic) cases and, if a single is locus is involved, penetrance less than $100 \%$ must be assumed. To make this adjustment Sherrington et al. (1988) explain that 'maximum likelihood estimates of penetrance were calculated'. This means that computer analyses were carried out assuming for each diagnostic model a range of possible penetrances. For each combination of model and penetrance a lod score was calculated and the highest thus produced was adopted. However, this manoeuvre of trying various models leads to an inflation of the maximum lod score and, as demonstrated by Weeks et al. (1990), 'the traditional limit of a lod score of 3 in the test for linkage is no longer appropriate, since a portion of the lod score is not due to linkage but merely to maximisation over models'. Weeks and colleagues (1990) also demonstrate a method of deflating such an apparent lod score by an appropriate proportion which they tentatively applied as closely as the data allows to the highest lod $(6.5)$ quoted by Sherrington et al. (1988) for their broad diagnostic model, and shows that it should be reduced to $5 \cdot 5$, and for their narrow (schizophrenia only) model from 4.98 to 3.46 . These omissions and ambiguities make it impossible to check the results reported by Sherrington et al. (1988) or to understand fully how they are derived from the data presented.

\section{ANOMALIES IN RESULTS REPORTED BY SHERRINGTON et al. (1988)}

There are in addition anomalies in the report of results which, to say the least, put their validity in doubt. These are in the areas of penetrance, 'fringe phenotypes' and linkage between markers. 
In some Mendelian disorders there may be individuals who carry the gene but do not manifest the phenotype. The proportion of such individuals among the gene carriers in a family defines the penetrance. In schizophrenia it is noticeable that some unaffected individuals have affected offspring and an affected parent, uncle, aunt or sib, which, if dominant transmission is assumed, must be ascribed to reduced penetrance. These individuals, although presumed to be gene carriers will, unless an allowance is made, count as unaffected in a linkage analysis and so weaken its power to detect linkage. An estimate of penetrance may be applied to the linkage analysis to compensate for this. The lower the estimate of penetrance, the greater the number of unaffected individuals who will be counted as carriers (i.e. carrying the schizophrenic gene) for the purpose of the linkage analysis. In Sherrington et al. (1988) penetrance was included in the computer program used for linkage, so that 'maximum likelihood estimates of penetrance were calculated', that is, the estimate of penetrance which gave the highest lod. These estimates were 86,76 and $73 \%$ (for the three analysis going from narrow to broader diagnostic criteria), Thus, a decrease of penetrance is shown when an increased number of phenotypes (i.e. cases presumed to be affected) was included in the analysis which is a reductio ad absurdum. Additionally, a remarkable feature of these pedigrees is that the proportion of affected relatives considerably exceeds that expected at $100 \%$ penetrance.

The populations used by Sherrington et al. (1988) for the three hierarchical analyses are, as previously described, graded from strictly defined schizophrenia to a more inclusive broader definition; the second and third groups each consist of the preceding group plus additional diagnoses (i.e. $1,2+1,3+2+1$ ). In the third group the additional cases, labelled 'fringe phenotypes' are a miscellaneous group of psychiatric disorders characterized by depression, neurosis and antisocial personality. Incorporation of these individuals increased the lod score from 5.2 to 7.4 (assuming $99 \%$ penetrance) (Brynjolfsson et al. 1989). The increase in lods from the first to the second group is surprising, but a further rise after the addition of 'fringe phenotypes' is frankly disjunctive. The implication of this finding is that a higher proportion of subjects with miscellaneous disorders without observable symptoms of schizophrenia than of subjects with schizophrenia show linkage to a marker for chromosome 5 which segregates with the putative gene for schizophrenia.

\section{MAPPING AROUND CHROMOSOME 5 DUPLICATED AREA}

In the family described by Basset (1988) the proband and uncle, who were thought to be schizophrenic, both possessed an extra copy of a small segment of chromosome $5(5 q 11-13)$ which was therefore thought to be causally connected with schizophrenia in these two subjects. Further mapping of this chromosomal segment has been carried out by Kennedy et al. (1988) and Gurling et al. (1989). Within this segment (5q11-5q13) Gurling et al. (1989) claim that 5 loci, identified by RFLP markers, have been mapped in relation to the postulated schizophrenia susceptibility locus. They therefore believe this locus lies between the loci identified by markers D5S76 and D5S39 for which Sherrington et al. (1988) claim cosegregation with schizophrenia sufficient to infer linkage between them. Also, between there two markers Kennedy et al. (1989) have mapped the locus for tyrosine hydroxylase and between them and D5S78 is the locus of a gene, important in nervous system development, labelled homeobox 2 (see Fig. 1). Gurling et al. (1989) find the same two markers (D5S76 and D5S39) linked to both tyrosine hydroxylase and D5S78, but, inexplicably, not to homeobox 2 which is nearer than D5S78. This is the equivalent of finding that a quantity is equal to each of two further quantities which, however, are not equal to each other.

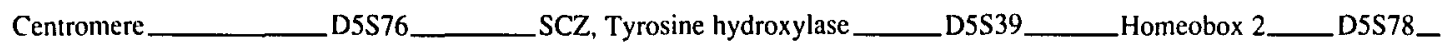

Fig. 1. Diagram showing the order of loci on the chromosomal segment $5 q 11-5 q 13$ according to the linkage analyses of Gurling et al. (1989) and Kennedy et al. (1989). SCZ = postulated schizophrenia susceptibility locus. 


\section{THE BASIS FOR SUSPECTING A SCHIZOPHRENIA SUSCEPTIBILITY GENE ON CHROMOSOME 5}

There is a more fundamental aspect to the question of what credence to give the postulate of a schizophrenia-susceptibility gene on chromosome 5 , arising from the fact that it rests wholly on the diagnoses of schizophrenia offered by Basset et al. (1988) on the two cases they report. The first case is a 20-year-old single male college student who, after being referred for investigation, was admitted to a psychiatric unit. School performance and social competence had been impaired for a number of years during which he had also shown a number of psychiatric symptoms without change of mood and without evidence of either mental retardation, drug disorder or alcoholic disorder. For 4 weeks before admission he experienced auditory hallucinations and delusions (paranoid and bizarre). On mental examination these symptoms were still present. ICD-9 (WHO, 1978) and DSMIII (APA, 1980) symptomatic criteria for schizophrenia were met. On physical examination he was found to be of small stature and had structural abnormalities affecting skull, face, eyes, ears, digits, toes, penis and internal organs. Following neuroleptic treatment, the florid symptoms subsided but low motivation, blunted emotions and poverty of speech remained. In a subsequent communication this young man and his uncle were reported to have abnormal eye tracking movements not present in his mother and two other relatives (Iacono et al. 1988). This brief account, the whole evidence supporting the diagnosis of schizophrenia, shows obvious gaps. What was the nature of the 'prodromal symptoms' and of the 'deterioration in social functioning and school performance' which preceded admission for several years? What previous investigations were carried out and what previous diagnosis made? How long before these cases were seen by Basset and colleagues had they been diagnosed as schizophrenia? Although the second patient had been under psychiatric care intermittently or continuously for over 30 years, the psychiatric features and history reported are similarly sparse. What is needed, as well as the assurances that standardized diagnostic criteria were met, is a full classical psychiatric history and systematic description of the mental state. Without this the statement that diagnostic criteria have been fulfilled amounts to a flat assertion of the diagnosis without supporting evidence. Valuable as they are, the limitations of standardized diagnostic criteria (Brockington et al. 1978) require that they are regarded as supporting not supplanting a full psychiatric history and examination as provided for instance, by Gottesman \& Shields (1972), who also provide blind independent diagnosis to guard against bias. Too much depends here upon diagnosis for it to be accepted on such a tenuous foundation.

\section{CONCLUSION}

It is obviously impossible to check the claim of Sherrington et al. (1988) from their published material or to understand the method by which their results have been obtained. The anomalies of their results are inexplicable in terms of their reported data. It must therefore be presumed that there is at present no strong evidence of linkage between a schizophrenia-susceptibility locus and a marker locus on chromosome 5.

Is it sensible to extend the search for linkage of markers to postulated heterogenous genes for schizophrenia susceptibility in the hope thereby of confirming their existence? Linkage analysis has so far been successful only in diseases showing Mendelian inheritance, which in most cases were due to a single locus. Such diseases usually appear early in life, have a low incidence and are characterized by a typical clinical picture, whereas schizophrenia appears in adult life, is common, poorly delineated and variable in manifestation. Propping \& Friedl (1988) point out that such common disorders have a complex aetiology where the explanatory power of genetic markers is very limited, as in coronary heart disease, where it is not the phenotype that follows Mendelian laws but the parameters relevant to its pathogenesis, such as lipoprotein polymorphism, and familial hypercholesterolaemia' acting with exogenous influences such as nutrition, smoking habits or stressful life-events'. Edwards (1990) has demonstrated the fundamental reasons for the inherently high improbability of detecting linkage if more than one gene is involved. Even such an apparently 
straightforward inborn error of metabolism as phenylketonuria, which has a clear Mendelian mode of inheritance and a relatively uniform phenotype is produced by more than one locus and by a disorder at any of several stages in the sequential pathway of the enzymes concerned (Scriver et al. 1989). It appears that in view of the confirmed negative results of linkage analysis in the genetic aspects of schizophrenia there is little utility in applying the method further until a candidate gene, as for instance the HLA complex and diabetes (for recent discussion see Charron, 1990), is available. Resources would be more usefully channelled into the investigation of parameters contributing to susceptibility of a disease as outlined by Propping \& Friedl (1988) in their model of common medical genetic disorders-drug response, gender response, smooth pursuit eye movements, body types, secondary or exogenous aetiology - and into the expansion of brain physiology and functional pathology.

D.C.WATT AND J.H.EDWARDS

The authors are indebted to Dr Pam Fain for valuable assistance in scrutiny of the pedigrees and other data presented in Sherrington et al. 1988.

\section{REFERENCES}

American Psychiatric Association (1980). Diagnostic and Statistical Manual of Mental Disorders (3rd ed). APA: Washington, DC.

Basset, A. S., McGillivray, B. C., Jones, B. D. \& Tapio Pantzer, J. (1988). Partial trisomy chromosome 5 cosegregating with schizophrenia. Lancet i, 799801.

Bateson, W. \& Punnet, R. G. (1908). Confirmation and extensions of Mendel's principles in other animals and plants. Report to the Evaluation Committee of the Royal Society. Saunders: London.

Bell, J. \& Haldane, J. B. S. (1937). The linkage between the genes for colour-blindness and haemophilia in man. Proceedings of the Royal Society of London 123, 119-150.

Brockington, I, F., Kendell, R. E. \& Left, J. P. (1978). Definitions of schizophrenia: concordance and prediction of outcome. Psychological Medicine 8, 387 - 398.

Brynjolfsson, J., Petursson, H., Sherrington, R. \& Gurling, H. (1989). Clinical variation in chromosome 5 linked schizophrenia. Abstracts of First World Congress on Psychiatric Genetics, Poster 26 , p. 95 , Cambridge.

Byerley, W. F. (1989). Genetic linkage revisited. Nature 340, 340-341.

Charron, D. (1990). Molecular basis of human linkage antigen class II disease associations. B Insulin-dependent diabetes mellitus. In Advances in Immunology, 48, (ed. F. J. Dixon), pp. 132-143. Academic Press: London.

Detera-Wadleigh, S. D., Goldin, L. R., Sherrington, R., Erio, I., Berretini, C. de M. W., Gurling, H. \& Gershon, E. S. (1989). Exclusion of linkage to $5 q 11-13$ in families with schizophrenia and other psychiatric disorders. Nature 340, 391-393.

Edwards, J. H. (1990). The linkage detection problem. Annals of Human Genetics 54, 253-275.

Egeland, J. A., Gerhard, D. S., Pauls, D. L., Sussex, J. N., Kidd, K. K., Allen, C. R., Hostetter, A. M. \& Housman, D. E. (1987). Bipolar affective disorders linked to DNA markers on chromosome II. Nature 325, 783-787.

Gottesman, I. I. \& Shields, J. (1972). Schizophrenia and Genetics. Academic Press: New York.

Gurling, H., Sherrington, R. P., Brynjolfsson, J., Read, T., Curtis, D., Baljindur, J., Potter, M. \& Petursson, H. (1989). Recent and future molecular genetic research into schizophrenia. Psychopharmacology' Bulletin 15, 373-382.

Haldane, J. B. S., Sprunt, A. D. \& Haldane, N. M. (1915). Reduplication in mice. Journal of Genetics 5, 133-135.

lacono, W. G., Basset, A. S. \& Jones, B. D. (1988). Eye-tracking dysfunction is associated with partial trisomy chromosome 5 and schizophrenia. Archives of General Psychiatry 45, 1140-41.

Kelsoe, J. R., Ginns, E. I., Egeland, J. E., Gerhard, D. S., Goldstein, A. M., Bale, S. J., Pauls, D. L., Long, R. T., Kidd, K. K., Conte,
G., Housman, D. E. \& Paul, S. M. (1989). Re-evaluation of the linkage relationship between chromosome $11 \mathrm{p}$ loci and the gene for bipolar affective disorder in the Old Older Amish. Nature $\mathbf{3 4 2}$, $238-243$.

Kennedy, J. L., Giuffra, L. A., Moises, H. W., Cavalli-Sforza, L. L., Pakotis, A. J., Kidd, J. R., Castiglione, C. M., Sjogren, B., Wetterberg, L. \& Kidd, K. K. (1988). Evidence against linkage of schizophrenia to markers on chromosome 5 in a northern Swedish pedigree. Nature 336, 167-170.

Lander, E. S. (1988). Splitting schizophrenia. Nature 336, 105-106.

Mohr, J. (1954). A study of linkage in man. Opera ex domo biologiae hereditariae humanae Universitatis Hafriensis 33. Munksgaard: Copenhagen.

Morgan, T. H. (1912). Further experiments with mutations in eyecolour of Drosophila: the loss of the orange factor. Journal of the Academy of Natural Sciences of Philadelphia 15, 321-346.

Morton, N. E. (1955). Sequential tests for the detection of linkage. American Journal of Human Genetics 7, 277-318.

Propping, P. \& Friedl, W. (1988). Genetic studies of biochemical, pathophysiological and pharmacological factors in schizophrenia. In Nosology. Epidemiology and Genetics of Schizophrenia, vol. 3 (ed. M. T. Tsuang and J. C. Simpson), ch. 25, pp. 579-608. In Handbook of Schizophrenia (ed. H. A. Nasrallah). Elsevier: Amsterdam.

Race, R. R. \& Sanger, R. (1975). Blood Groups in Man (6th edn). Blackwell: Oxford.

Scriver, C. R., Kaufman, S., Woo, S. L. C. (1989). The hyperphenylalaninaemias, Vol. 1. ch. 15, pp. 495-546. In The Metabolic Basis of Inherited Disease (6th edition) (ed. C. R. Scriver, A. L. Baudet, W. S. Sly, Valle, D.). McGraw Hill: New York.

Sherrington, R., Brynjolfsson, H. P., Potter, M., Pudleston, K., Barraclough, B., Wasmuth, J., Dobbs, M. \& Gurling, H. (1988). Localisation of a susceptibility locus for schizophrenia on chromosome 5. Nature 336, 164-167.

St. Clair, D., Blackwood, D., Muir, W., Baillie, D., Hubbard, A., Wright, A. \& Evans, H. J. (1989). No linkage of chromosome 5q l l-q 13 markers to schizophrenia in Scottish families. Nature 339, 305-309.

Sturt, E. \& McGuffin, P. (1985). Can linkage and marker association resolve the genetic aetiology of psychiatric disorders? Review and argument. Psychological Medicine 15, 455 462.

Weeks, D. E., Lehnor, T., Squires-Wheeler, E., Kaufmann, C. \& Ott, J. (1990). Measuring the inflation of the lod score due to its maximation over model parameter values in human linkage analysis. Genetic Epidemiology 7, 237-243.

World Health Organization (1978). Mental Disorders: Glossary and Guide to Their Classification in Accordance with the Ninth Revision of the International Classification of Diseases. WHO: Geneva. 